\title{
ON THE BEHAVIOR OF CLAMPED PLATES UNDER LARGE COMPRESSION*
}

\author{
P. R. S. ANTUNES ${ }^{\dagger}$, D. BUOSO $\ddagger$, AND P. FREITAS ${ }^{\S}$
}

\begin{abstract}
We determine the asymptotic behavior of eigenvalues of clamped plates under large compression by relating this problem to eigenvalues of the Laplacian with Robin boundary conditions. Using the method of fundamental solutions, we then carry out a numerical study of the extremal domains for the first eigenvalue, from which we see that these depend on the value of the compression, and start developing a boundary structure as this parameter is increased. The corresponding number of nodal domains of the first eigenfunction of the extremal domain also increases with the compression.
\end{abstract}

Key words. biharmonic operator, plate with tension, plate with compression, eigenvalues, asymptotics, extremal domains

AMS subject classifications. Primary, 35J30; Secondary, 35P15, 35P20, 49R50, 74K20

DOI. $10.1137 / 19 \mathrm{M} 1249606$

1. Introduction. Let $\Omega$ be a smooth bounded domain in $\mathbb{R}^{N}, N \geq 2$. We are interested in the following eigenvalue problem:

$$
\begin{cases}\Delta^{2} u+\alpha \Delta u=\lambda u & \text { in } \Omega, \\ u=\frac{\partial u}{\partial \nu}=0 & \text { on } \partial \Omega,\end{cases}
$$

considered as a model for a clamped plate. Here $\alpha$ is a real parameter corresponding to the quotient between the tension and the flexural rigidity and, depending on its sign, represents whether the plate is under tension $(\alpha<0)$ or compression $(\alpha>0)$. For domains $\Omega$ as described above, the eigenvalues of (1.1) form an infinite sequence

$$
\lambda_{1} \leq \lambda_{2} \leq \cdots \leq \lambda_{k} \leq \cdots,
$$

where $\lambda_{k}=\lambda_{k}(\Omega, \alpha)$ approaches $+\infty$ as $k$ goes to infinity.

The study of this and similar problems has been considered in the literature continuously over time since the works of Lord Rayleigh [26] and Love [21] on clamped plates. We refer the reader to the book [16] for an extensive historical and scientific overview on the mechanics of plates through the Kirchhoff-Love model, which leads to problem (1.1).

In this paper, we are concerned with two issues related to (1.1), namely the asymptotic behavior of the eigenvalues $\lambda_{k}$ as the parameter $\alpha$ approaches $+\infty$ (the

* Received by the editors March 12, 2019; accepted for publication (in revised form) July 10, 2019; published electronically September 24, 2019.

https://doi.org/10.1137/19M1249606

Funding: This work was partially supported by the Fundação para a Ciência e a Tecnologia (Portugal) through the program "Investigador FCT" with reference IF $/ 00177 / 2013$ and the project Extremal spectral quantities and related problems (PTDC/MAT-CAL/4334/2014).

†Secção de Matemática, Departmento de Ciências e Tecnologia, Universidade Aberta, Palácio Ceia, 1269-001 Lisbon, Portugal, and Grupo de Física Matemática, Faculdade de Ciências, Universidade de Lisboa, Campo Grande, Edifício C6, P-1749-016 Lisboa, Portugal (prantunes@fc.ul.pt).

${ }_{\ddagger}^{\ddagger}$ EPFL, SB MATH SCI-SB-JS, Station 8, CH-1015 Lausanne, Switzerland (davide.buoso@epfl.ch).

§Departamento de Matemática, Instituto Superior Técnico, Universidade de Lisboa, P-1049-001 Lisboa, Portugal, and Grupo de Física Matemática, Faculdade de Ciências, Universidade de Lisboa, Campo Grande, Edifício C6, P-1749-016 Lisboa, Portugal (psfreitas@fc.ul.pt). 
case of $-\infty$ was considered in [14]) and the extremal domains of such eigenvalues as $\alpha$ varies. In the first instance, the above problem is closely related to

$$
\begin{cases}\Delta^{2} v+a v+\gamma \Delta v=0 & \text { in } \Omega, \\ v=\frac{\partial v}{\partial \nu}=0 & \text { on } \partial \Omega,\end{cases}
$$

where now the eigenvalue parameter is $\gamma=\gamma(a)$, and the (positive) parameter $a$ stands for the elasticity constant of the medium surrounding the plate. We know from a result in [19] that for $(1.2)$

$$
\lim _{a \rightarrow+\infty} \frac{\gamma_{1}(a)}{\sqrt{a}}=2
$$

which when translated into the eigenvalue problem in (1.1) yields

$$
\lim _{\alpha \rightarrow+\infty} \frac{\lambda_{1}(\alpha)}{\alpha^{2}}=-\frac{1}{4} .
$$

Our main result along these lines is to extend this to all eigenvalues $\lambda_{k}$. This is achieved by an approach different from that used in [19], involving now a connection (which, to the best of our knowledge, is new) between the eigenvalues of the clamped plate problem (1.1) and those of a Robin eigenvalue problem for the Dirichlet Laplacian in the case where $\Omega$ is a ball; see section 3 for the details. To be more precise, we prove the following.

THEOREM 1.1 (asymptotic behavior of the $k$ th eigenvalue). Let $\Omega$ be a bounded domain in $\mathbb{R}^{N}$. Then, for any positive integer $k$, the eigenvalues of (1.1) satisfy

$$
\lambda_{k}(\Omega, \alpha)=-\frac{\alpha^{2}}{4}+\mathrm{o}\left(\alpha^{2}\right)
$$

as $\alpha \rightarrow+\infty$. Moreover,

$$
\lambda_{1}(\Omega, \alpha)=-\frac{\alpha^{2}}{4}+\mathrm{O}(\alpha)
$$

as $\alpha \rightarrow+\infty$.

For positive values of $\alpha$, each of the eigenvalue curves $\lambda_{k}=\lambda_{k}(\alpha)$ is, in fact, made up of analytic eigenvalue branches which intersect each other; see Figure 1, where to illustrate this effect we plotted the quantity $\lambda_{k}(\Omega, \alpha)+\frac{\alpha^{2}}{4}$ for the disk and for ellipses. This branch-switching phenomenon makes it much more difficult to obtain further terms in the asymptotic expansion, and it is the independence of the first term on the order of the eigenvalue which allows us to derive the expansion for all $k$. In the particular case where $\Omega$ is a ball of radius $R$, which is at the heart of the proof of Theorem 1.1, we are able to prove that the number of such eigenvalue branches which make up the $k$ th eigencurve is finite, and we determine further terms in the asymptotic expansion of these analytic branches. These results are summarized in the following.

THEOREM 1.2 (asymptotic behavior of analytic eigenvalue branches for balls). For any analytical branch of the eigenvalues of problem (1.1) when $\Omega$ is a ball of radius $R$, we have

$$
\lambda=-\frac{\alpha^{2}}{4}+\frac{c_{1} \alpha}{R^{2}}+\frac{c_{2}}{R^{4}}+\mathrm{o}(1)
$$


(a)
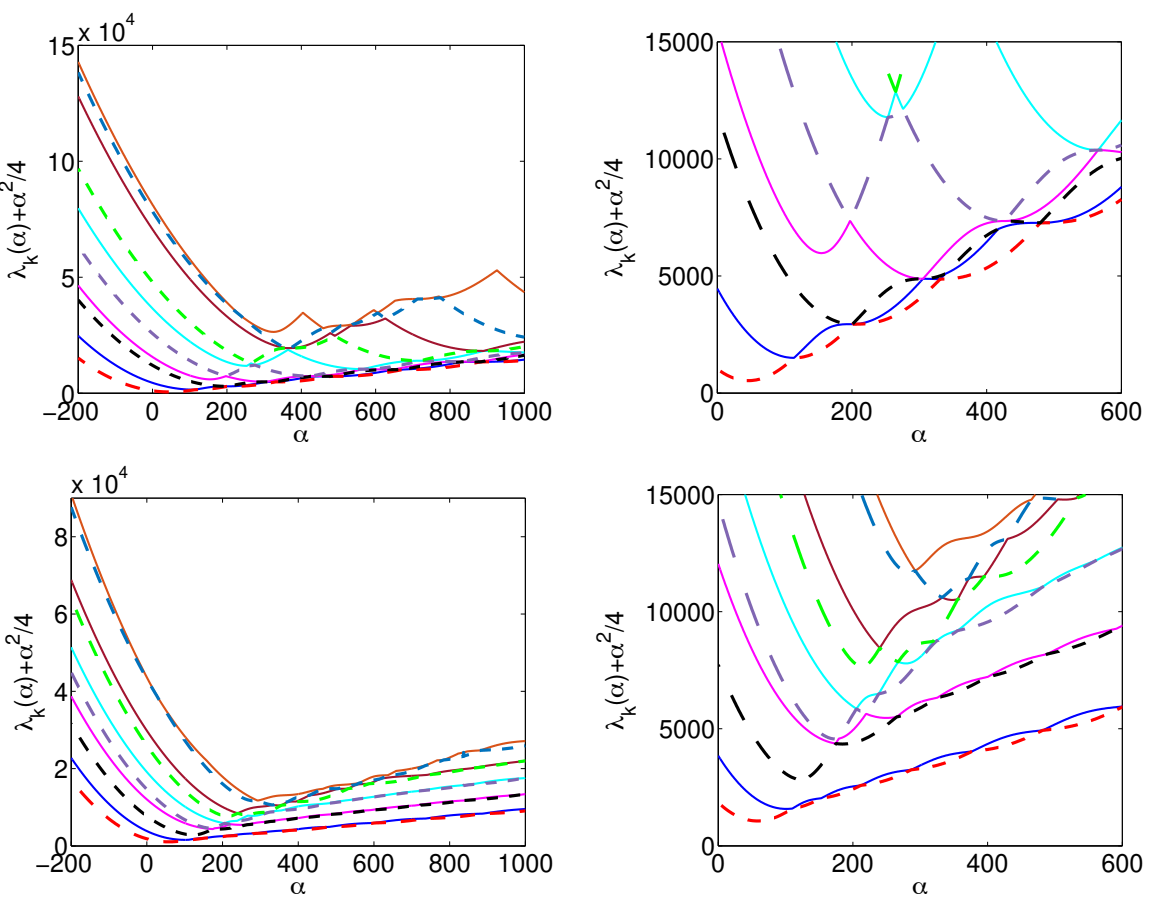

FIG. 1. (a) Plot of the quantities $\lambda_{k}(\Omega, \alpha)+\frac{\alpha^{2}}{4}, k=1,2, \ldots, 10$, for the disk with unit area, for $\alpha \in[-200,1000]$ (left plot) and a zoom for $\alpha \in[0,600]$, illustrating the behavior of the smallest eigenvalues as a function of $\alpha$ (right plot). (b) Similar results for an ellipse with unit area and eccentricity equal to $\sqrt{3} / 2$.

as $\alpha \rightarrow+\infty$, where $c_{1}$ and $c_{2}$ are constants depending on the eigenvalue branch, with $c_{1}$ being positive. In the case of the first eigenvalue, we have

$$
\lambda_{1}=-\frac{\alpha^{2}}{4}+\frac{\pi^{2} \alpha}{2 R^{2}}+\frac{\pi^{2}\left(N^{2}-1-\pi^{2}\right)}{4 R^{4}}+\mathrm{o}(1) .
$$

The full description of the coefficients $c_{1}$ and $c_{2}$ may be found in Theorem 3.3 in section 3 .

It is possible to consider problem (1.1) with other boundary conditions, such as the Navier setting. This is not as interesting from a mathematical perspective since the problem then reduces directly to the study of the second-order elliptic operator $\Delta+\alpha / 2$. However, and as we show in section 4 , there is a major difference between the Dirichlet and Navier cases in that for the Navier problem the number of crossings of analytic branches to make up an eigencurve corresponding to the $k$ th eigenvalue is actually infinite for each $k$. Complex crossing and avoided-crossing patterns seem to be a characteristic of such systems in the large compression regime, and they have also been identified in the one-dimensional fourth-order problem with different boundary conditions studied in [11].

Concerning our second topic of study, namely extremal domains for eigenvalues of problem (1.1), even in the case where the parameter $\alpha$ vanishes the problem is known to be extremely difficult with results available only in two and three dimensions (see $[22,5]$, respectively); for $(1.2)$, there are no complete results in any dimension. 
Once $\alpha$ is taken to be nonzero in (1.1), the only existing result is an extension to sufficiently small positive values of $\alpha$ in two dimensions [4]. Our purpose in this part is thus mainly to provide a numerical exploration of the different types of extremal domains under an area restriction, showing in particular that the ball is no longer a minimizer for large compression.

We consider the numerical solution of the eigenvalue problem (1.1) using the Method of Fundamental Solutions (see, e.g., [1, 3]). This is a meshless numerical method where the approximation is made by a discretization of an expansion in terms of the single and double layer potentials. In particular, by construction, the numerical approximation satisfies the fourth-order partial differential equation and we can focus just on the approximation of the boundary conditions of the problem. The computational implementation of this numerical method is described in section 6.1 , and some numerical results for the shape optimization problem are presented in section 6.3 . In particular, we will study minimizers of the first eigenvalue of problem (1.1) subject to an area constraint. The obtained numerical results suggest that the minimizer depends on the parameter $\alpha$, with the ball being the minimizer for all negative $\alpha$ and then extending to $\alpha \in\left[0, \alpha^{\star}\right]$ for some positive $\alpha^{\star}$. Note that this last result corresponds to that proved in [4] for sufficiently small $\alpha$, with our numerical simulations suggesting that, in fact, one may take $\alpha^{\star}$ to be at least as large as the first buckling eigenvalue. For large values of the parameter $\alpha$, we obtain some nontrivial minimizers; see Figure 3.

This numerical study has been performed mainly among general simply connected domains. However, we performed also the optimization of the first eigenvalue of problem (1.1) among annuli having unit area and compared the optimal values that were obtained with the corresponding values of the ball. These results suggest that the first eigenvalue of the disk is always smaller than the corresponding eigenvalue of the optimal annulus, independently of the parameter $\alpha$.

2. Statement of the problem. We start by observing that problem (1.1) has the following weak formulation:

$$
\int_{\Omega} \Delta u \Delta \phi-\alpha \nabla u \nabla \phi=\lambda \int_{\Omega} u \phi \quad \forall \phi \in H_{0}^{2}(\Omega),
$$

and its eigenvalues may be described through their variational characterizations:

$$
\lambda_{k}^{D}(\Omega, \alpha)=\min _{\substack{V \subset H_{0}^{2}(\Omega) \\ \operatorname{dim} V=k}} \max _{0 \neq u \in V} \frac{\int_{\Omega}(\Delta u)^{2}-\alpha|\nabla u|^{2}}{\int_{\Omega} u^{2}} .
$$

In what follows, whenever the meaning is clear from the context, we will drop either argument in $\lambda_{k}^{D}(\Omega, \alpha)$ for the sake of simplicity.

In order to determine the eigenfunctions of problem (1.1) when $\Omega=B_{R}(0)$, we rewrite (1.1) as

$$
\left(\Delta+\alpha_{+}\right)\left(\Delta+\alpha_{-}\right) u=0
$$

where

$$
\alpha_{+}=\frac{\alpha}{2}+\sqrt{\frac{\alpha^{2}}{4}+\lambda}, \quad \alpha_{-}=\frac{\alpha}{2}-\sqrt{\frac{\alpha^{2}}{4}+\lambda} .
$$

Both $\alpha_{+}$and $\alpha_{-}$are always real, as may be seen from inequality (4.6), and $\alpha_{+}$is always positive while the sign of $\alpha_{-}$depends on the sign of the eigenvalue $\lambda$. 
For positive $\lambda$, it is known that the solution of (2.3) can be written as (cf. [5])

$$
u(r, \theta)=r^{1-\frac{N}{2}}\left[A J_{k+\frac{N}{2}-1}\left(r \sqrt{\alpha_{+}}\right)+B I_{k+\frac{N}{2}-1}\left(r \sqrt{-\alpha_{-}}\right)\right] S_{k}(\theta),
$$

where $J_{\nu}$ and $I_{\nu}$ are the Bessel and the modified Bessel functions, respectively, of the first kind of order $\nu$, and $S_{k}$ are the spherical harmonic functions of order $k$. The boundary conditions then yield the following system of equations:

$$
\left\{\begin{array}{l}
A f_{k}(R)+B g_{k}(R)=0 \\
A f_{k}^{\prime}(R)+B g_{k}^{\prime}(R)=0
\end{array}\right.
$$

where we have set

$$
f_{k}(r)=r^{1-\frac{N}{2}} J_{k+\frac{N}{2}-1}\left(r \sqrt{\alpha_{+}}\right) \quad \text { and } \quad g_{k}(r)=r^{1-\frac{N}{2}} I_{k+\frac{N}{2}-1}\left(r \sqrt{-\alpha_{-}}\right) .
$$

Since we are interested in the existence of nontrivial solutions of system (2.6), we impose the corresponding determinant to be zero, namely

$$
f_{k}(R) g_{k}^{\prime}(R)-g_{k}(R) f_{k}^{\prime}(R)=0,
$$

from which we obtain the corresponding eigenvalues and, as a consequence, the general form of the eigenfunctions. Using standard Bessel function identities, (2.7) may be rewritten as

$$
\begin{aligned}
& \frac{R J_{k+\frac{N}{2}-1}\left(R \sqrt{\alpha_{+}}\right)}{k J_{k+\frac{N}{2}-1}\left(R \sqrt{\alpha_{+}}\right)-R \sqrt{\alpha_{+}} J_{k+\frac{N}{2}}\left(R \sqrt{\alpha_{+}}\right)} \\
& =\frac{R I_{k+\frac{N}{2}-1}\left(R \sqrt{-\alpha_{-}}\right)}{k I_{k+\frac{N}{2}-1}\left(R \sqrt{-\alpha_{-}}\right)+R \sqrt{-\alpha_{-}} I_{k+\frac{N}{2}}\left(R \sqrt{-\alpha_{-}}\right)} .
\end{aligned}
$$

When $\lambda$ is strictly negative, $\alpha_{-}$is strictly positive and, in place of $(2.5)$, we now have

$$
u(r, \theta)=r^{1-\frac{N}{2}}\left[A J_{k+\frac{N}{2}-1}\left(r \sqrt{\alpha_{+}}\right)+B J_{k+\frac{N}{2}-1}\left(r \sqrt{\alpha_{-}}\right)\right] S_{k}(\theta),
$$

where the coefficients are given by a system similar to (2.6), and the eigenvalues are now solutions of

$$
\begin{aligned}
& \frac{J_{k+\frac{N}{2}-1}\left(R \sqrt{\alpha_{+}}\right)}{k R J_{k+\frac{N}{2}-1}\left(R \sqrt{\alpha_{+}}\right)-\sqrt{\alpha_{+}} J_{k+\frac{N}{2}}\left(R \sqrt{\alpha_{+}}\right)} \\
& =\frac{J_{k+\frac{N}{2}-1}\left(R \sqrt{\alpha_{-}}\right)}{k R J_{k+\frac{N}{2}-1}\left(R \sqrt{\alpha_{-}}\right)-\sqrt{\alpha_{-}} J_{k+\frac{N}{2}}\left(R \sqrt{\alpha_{-}}\right)} .
\end{aligned}
$$

Finally, it remains to consider the case $\lambda=0$, which behaves in a slightly different way. We note that then $\alpha_{+}=\alpha$ while $\alpha_{-}=0$, and in particular this means that $\alpha$ has to be an eigenvalue of the following buckling problem:

$$
\begin{cases}\Delta^{2} u=-\Lambda \Delta u & \text { in } \Omega, \\ u=\frac{\partial u}{\partial \nu}=0 & \text { on } \partial \Omega,\end{cases}
$$

for which the eigenfunctions are known to be of the form (they can be derived in a similar way as for the other cases)

$$
u(r, \theta)=\left[A r^{1-\frac{N}{2}} J_{k+\frac{N}{2}-1}(r \sqrt{\alpha})+B r^{k}\right] S_{k}(\theta) .
$$


In particular, $\alpha$ has to be a solution of the following:

$$
J_{k+\frac{N}{2}}(R \sqrt{\alpha})=0 .
$$

Moreover, if $\alpha$ is the $k$ th eigenvalue $\Lambda_{k}$ of the buckling problem (2.11), we immediately deduce that the vanishing eigenvalue of problem (1.1) is exactly the $k$ th one $\lambda_{k}$, and the multiplicity will be the same of $\Lambda_{k}$.

3. Connection to the Robin Laplacian. Even though there are no simple relations between the Laplacian and the Bilaplacian in general (apart from the Navier problem (4.1)), if we consider the generic situation of problem (1.1) in the ball in $\mathbb{R}^{N}$ with $\alpha \in \mathbb{R}$, we can draw a very precise connection to the Robin Laplacian.

To this end, we recall that the Robin problem for the Laplace operator is as follows:

$$
\begin{cases}-\Delta u=\sigma u & \text { in } \Omega \\ \frac{\partial u}{\partial \nu}+\beta u=0 & \text { on } \partial \Omega\end{cases}
$$

For any real value of $\beta$, the corresponding spectrum consists of a nondecreasing sequence of eigenvalues with finite multiplicities diverging to plus infinity. In particular, for positive values of $\beta$ the eigenvalues are all strictly positive, while for $\beta=0$ the Robin problem (3.1) becomes the Neumann problem. It is also known that, as $\beta \rightarrow+\infty$, problem (3.1) converges to the Dirichlet problem for the Laplace operator, namely

$$
\begin{cases}-\Delta u=\gamma u & \text { in } \Omega, \\ u=0 & \text { on } \partial \Omega\end{cases}
$$

whose eigenvalues we will denote by

$$
0<\gamma_{1} \leq \gamma_{2} \leq \cdots \rightarrow+\infty
$$

We recall that the eigenfunctions of (3.1) on a ball can be sorted out into three categories (cf. section 2):

i. if the eigenvalue $\sigma$ is positive, the eigenfunction is of the form

$$
r^{1-\frac{N}{2}} J_{k+\frac{N}{2}-1}(r \sqrt{\sigma}) S_{k}(\theta),
$$

and eigenvalues are solutions of

$$
\left(\frac{k}{R}+\beta\right) J_{k+\frac{N}{2}-1}(R \sqrt{\sigma})=\sqrt{\sigma} J_{k+\frac{N}{2}}(R \sqrt{\sigma})
$$

ii. if the eigenvalue $\sigma$ is negative, the eigenfunction is of the form

$$
r^{1-\frac{N}{2}} I_{k+\frac{N}{2}-1}(r \sqrt{-\sigma}) S_{k}(\theta),
$$

and eigenvalues are solutions of

$$
\left(\frac{k}{R}+\beta\right) I_{k+\frac{N}{2}-1}(R \sqrt{-\sigma})=-\sqrt{-\sigma} I_{k+\frac{N}{2}}(R \sqrt{-\sigma})
$$

iii. if the eigenvalue $\sigma$ is zero, the eigenfunction is of the form $r^{k} S_{k}(\theta)$, and in particular this occurs when $\beta=-\frac{k}{R}$. 
At this point, it is clear that any eigenfunction of the clamped plate problem (1.1) on the ball can be thought of as the sum of two different eigenfunctions of the Robin problem for the Laplacian (3.1). A first condition that these two Robin eigenfunctions have to satisfy is that their spherical parts coincide. This implies that they must come from two different eigenvalues; in particular, we have that these two eigenvalues are $\alpha_{+}$and $\alpha_{-}$, and the multiplicities must coincide. Furthermore, such eigenfunctions must have the same index $\nu=k+\frac{N}{2}-1$ in their Bessel function part. Let us call $v_{1}, v_{2}$ two such eigenfunctions (with associated eigenvalues $\sigma_{1}, \sigma_{2}$ ), and let

$$
v_{j}(r, \theta)=v_{j}^{R}(r) S(\theta) ;
$$

i.e., we denote by $v_{j}^{R}$ the radial part. Since we want the boundary conditions in the clamped plate problem (1.1) to be satisfied, the only way to combine $v_{1}$ and $v_{2}$ is to set

$$
u=v_{1}^{R}(R) v_{2}-v_{2}^{R}(R) v_{1},
$$

as can be easily checked from the boundary conditions in the Robin problem for the Laplace operator (3.1). In particular, $v_{1}$ and $v_{2}$ must be Robin eigenfunctions associated with the same parameter $\beta$. As for the equation, we observe that

$$
\Delta^{2} v_{j}+\alpha \Delta v_{j}=\left(\sigma_{j}^{2}-\alpha \sigma_{j}\right) v_{j}
$$

and the equality $\sigma_{1}^{2}-\alpha \sigma_{1}=\sigma_{2}^{2}-\alpha \sigma_{2}$ is naturally satisfied since

$$
\alpha=\alpha_{+}+\alpha_{-}=\sigma_{1}+\sigma_{2}, \quad \lambda=-\alpha_{+} \alpha_{-}=-\sigma_{1} \sigma_{2} .
$$

On the other hand, letting $\beta$ go to infinity we get that, for specific values of $\alpha$ and $\lambda$, we should consider eigenvalues of the Dirichlet Laplacian (3.2) instead. From the literature (see, e.g., [8] and the references therein, and also [15] for a study of the first two Robin eigenvalues), we know that all the analytical branches related to the same Bessel function $J_{k+\frac{N}{2}-1}\left(I_{k+\frac{N}{2}-1}\right.$ when the eigenvalue is negative, $r^{k}$ if zero) can be continued at $\beta=\infty$ generating a function which wraps around $\mathbb{R}$ infinitely many times. If we call $\sigma_{k, j}(\beta)$ the $j$ th eigenvalue associated with $J_{k+\frac{N}{2}-1}$, then the analytical branches of eigenvalues of problem (1.1) are given by

$$
-\sigma_{k, j}(\beta) \sigma_{k, j+t}(\beta)
$$

for some $t \in \mathbb{N}$, where the parameter $j$ is of no relevance here since any time $\beta$ reaches infinity $j$ has to be replaced by $j+1$ as

$$
\gamma_{k, j}=\sigma_{k, j}(+\infty)=\sigma_{k, j+1}(-\infty),
$$

where $\gamma_{k, j}$ is the $j$ th eigenvalue of the Dirichlet problem for the Laplace operator (3.2) associated with $J_{k+\frac{N}{2}-1}$. In particular, different branches of eigenvalues of the clamped plate problem (1.1) associated with the Bessel index $k+\frac{N}{2}-1$ are indexed by the parameter $t$ in (3.5). We remark that all the branches are of this type, and hence no other branches are present. We sum up all these arguments in the following.

TheOREM 3.1. Let $\beta \in \mathbb{R}$, and let $v_{1}$ and $v_{2}$ be any two eigenfunctions of problem (3.1) in a ball $B_{R}(0)$ associated with the eigenvalues $\sigma_{1}$ and $\sigma_{2}$, respectively, and having the same spherical part, namely

$$
v_{j}(x)=v_{j}^{R}(r) S(\theta), \quad j=1,2 .
$$


Then the function defined in (3.3) is an eigenfunction of problem (1.1) in the ball $B_{R}(0)$ associated with the eigenvalue $\lambda=-\sigma_{1} \sigma_{2}$ and with the parameter $\alpha=\sigma_{1}+\sigma_{2}$.

This representation completely characterizes the analytic branch of the eigenvalue $\lambda=-\sigma_{1} \sigma_{2}$ (for $\alpha \in \mathbb{R}$ ) as the parameter $\beta$ varies. In the limits $\beta \rightarrow \pm \infty$, we have that the eigenvalue $\lambda$ can be written as a product of eigenvalues of the Dirichlet problem for the Laplace operator (3.2), and the corresponding eigenfunction can also be written as a combination of eigenfunctions of problem (3.2).

In addition, all analytic branches of eigenvalues of the clamped plate problem (1.1) can be represented in this fashion.

Theorem 3.1 allows us to study the behavior of the eigenvalues as $\alpha= \pm \infty$. Actually, for the case $\alpha=-\infty$, the convergence is well known in the literature for any smooth domain (see, e.g., [14, p. 392]).

TheOREM 3.2. Let $w_{\alpha}$ be the eigenfunction associated with $\lambda_{k}(\alpha)$, and suppose that there exists a point $\alpha_{0} \in \mathbb{R}$ such that $w_{\alpha} \in C^{5}(\Omega)$ for any $\alpha<\alpha_{0}$. Then

$$
\lambda_{k}(\alpha)=-\alpha \gamma_{k}+\sqrt{-\alpha} \int_{\partial \Omega}\left|\nabla u_{k}\right|^{2}+\mathrm{O}(1)
$$

as $\alpha \rightarrow-\infty$, where $u_{k}$ is an eigenfunction of the Dirichlet problem for the Laplace operator (3.2) associated with $\gamma_{k}$.

We recall that, thanks to classical regularity theory for elliptic operators (cf. [16]), if $\Omega \in C^{5, \delta}$, then $w_{\alpha} \in C^{5, \delta}(\Omega)$ for any $\alpha \in \mathbb{R}$ and, in particular, balls satisfy the hypotheses of Theorem 3.2. It is easily seen then that we can recover the first term of the asymptotics (3.6) using the known asymptotics for the Robin problem (see [25] and the references therein).

Regarding the asymptotics as $\alpha \rightarrow+\infty$, we compute it using the knowledge that for any given branch when we get to $\beta=\infty$ we obtain that both $\alpha$ and $\lambda$ can be expressed in terms of zeros of Bessel functions:

$$
\alpha=\frac{j_{k+\frac{N}{2}-1, m}^{2}+j_{k+\frac{N}{2}-1, m+t}^{2}}{R^{2}}, \quad \lambda=-\frac{j_{k+\frac{N}{2}-1, m}^{2} \times j_{k+\frac{N}{2}-1, m+t}^{2}}{R^{4}},
$$

where $j_{\nu, m}$ is the $m$ th zero of $J_{\nu}$, whose asymptotic behavior is known to be (cf. [23, formula (10.21.19)])

$$
j_{\nu, m} \sim\left(m+\frac{\nu}{2}-\frac{1}{4}\right) \pi-\frac{4 \nu^{2}-1}{8\left(m+\frac{\nu}{2}-\frac{1}{4}\right) \pi}+\mathrm{o}\left(\frac{1}{m^{2}}\right)
$$

as $m \rightarrow \infty$.

Let us now denote by $\psi_{m}$ and $\psi_{m+t}$ two eigenfunctions of the Dirichlet problem for the Laplace operator (3.2) associated with $\gamma_{m}=R^{-2} j_{k+\frac{N}{2}-1, m}^{2}$ and $\gamma_{m+t}=$ $R^{-2} j_{k+\frac{N}{2}-1, m+t}^{2}$, respectively, having the same spherical part and normalized such that $\psi_{m}+\psi_{m+t}$ is an eigenfunction of the clamped plate problem (1.1) under condition (3.7). Then, using the Rayleigh quotient representation of $\lambda$, we have

$$
\begin{aligned}
\lambda & =-\frac{\alpha^{2}}{4}+\frac{\int_{B_{R}}\left[\Delta\left(\psi_{m}+\psi_{m+t}\right)+\frac{\alpha}{2}\left(\psi_{m}+\psi_{m+t}\right)\right]^{2}}{\int_{B_{R}}\left(\psi_{m}+\psi_{m+t}\right)^{2}} \\
& =-\frac{\alpha^{2}}{4}+\left(\frac{\gamma_{m}-\gamma_{m+t}}{2}\right)^{2} .
\end{aligned}
$$


We recall that in this particular case we have $\alpha=\left(j_{\nu, m}^{2}+j_{\nu, m+t}^{2}\right) R^{-2}$, where we set $\nu=k+\frac{N}{2}-1$ for simplicity. We now compute the asymptotics for the remainder in (3.9) and get

$$
\begin{aligned}
\frac{\left(j_{\nu, m}^{2}-j_{\nu, m+t}^{2}\right)^{2}}{4 R^{2}\left(j_{\nu, m}^{2}+j_{\nu, m+t}^{2}\right)} & \approx \frac{\left[\left(m+\frac{\nu}{2}-\frac{1}{4}\right)^{2} \pi^{2}-\left(m+t+\frac{\nu}{2}-\frac{1}{4}\right)^{2} \pi^{2}\right]^{2}}{4 R^{2}\left[\left(m+\frac{\nu}{2}-\frac{1}{4}\right)^{2} \pi^{2}+\left(m+t+\frac{\nu}{2}-\frac{1}{4}\right)^{2} \pi^{2}\right]} \\
& \approx \frac{t^{2} \pi^{2}}{2 R^{2}},
\end{aligned}
$$

telling us that $\lambda \sim-\frac{\alpha^{2}}{4}+\frac{\alpha t^{2} \pi^{2}}{2 R^{2}}+\mathrm{o}(\alpha)$. Going further, we can get

$$
\begin{aligned}
\frac{\left(j_{\nu, m}^{2}-j_{\nu, m+t}^{2}\right)^{2}}{4 R^{4}}- & \frac{\left(j_{\nu, m}^{2}+j_{\nu, m+t}^{2}\right) t^{2} \pi^{2}}{2 R^{4}} \\
\approx \frac{1}{4 R^{4}} & {\left[\left(m+\frac{\nu}{2}-\frac{1}{4}\right)^{2} \pi^{2}-\left(m+t+\frac{\nu}{2}-\frac{1}{4}\right)^{2} \pi^{2}\right]^{2} } \\
& \quad-\frac{t^{2} \pi^{4}}{2 R^{4}}\left[\left(m+\frac{\nu}{2}-\frac{1}{4}\right)^{2}+\left(m+t+\frac{\nu}{2}-\frac{1}{4}\right)^{2}-\frac{4 \nu^{2}-1}{2 \pi^{2}}\right] \\
\approx & \frac{t^{2} \pi^{2}\left(4 \nu^{2}-1-t^{2} \pi^{2}\right)}{4 R^{4}},
\end{aligned}
$$

and hence we have the following.

THEOREM 3.3. For any analytical branch of the eigenvalues of problem (1.1) on a ball $B_{R}$ of radius $R$, we have

$$
\lambda=-\frac{\alpha^{2}}{4}+\frac{\alpha t^{2} \pi^{2}}{2 R^{2}}+\frac{t^{2} \pi^{2}\left(4 \nu^{2}-1-t^{2} \pi^{2}\right)}{4 R^{4}}+\mathrm{o}(1)
$$

as $\alpha \rightarrow+\infty$, where $\nu=k+\frac{N}{2}-1$ is the index of the associated Bessel functions, and $t$ is the parameter introduced in (3.5).

We observe that, even if at a first glance the presence of the parameter $t$ may seem unnatural, it may be compared, for example, with the ordering number for zeros of Bessel functions $j_{\nu, k}$. From this perspective, it is natural that it appears in formula (3.10).

We are now ready to prove Theorem 1.1.

Proof of Theorem 1.1. In the case of a general domain $\Omega$, we shall denote the radius of the largest inscribed ball and that of the smallest ball containing $\Omega$ by $R_{i}, R_{c}$, respectively. By the inclusion properties for problem (1.1), we know that any eigenvalue of $\Omega$ is bounded from above and from below by the corresponding eigenvalues of the inscribed and circumscribed balls, respectively. This immediately proves (1.3). For higher eigenvalues, it will, in general, be difficult to determine the precise order of each eigenvalue, but in the case of the first eigenvalue it is possible to identify the corresponding branch, namely that obtained by making $t=1$ and $k=0$, and in turn obtain the following (asymptotic) expression:

$$
-\frac{\alpha^{2}}{4}+\frac{\alpha \pi^{2}}{2 R_{c}^{2}} \lesssim \lambda_{1}(\Omega) \lesssim-\frac{\alpha^{2}}{4}+\frac{\alpha \pi^{2}}{2 R_{i}^{2}},
$$


which implies (1.4).

4. The Navier problem. We now turn our attention to the following eigenvalue problem:

$$
\begin{cases}\Delta^{2} u+\alpha \Delta u=\lambda u & \text { in } \Omega \\ u=\Delta u=0 & \text { on } \partial \Omega\end{cases}
$$

for any $\alpha \in \mathbb{R}$. We immediately notice the resemblance of the Navier problem (4.1) with problem (1.1), as its weak formulation reads as

$$
\int_{\Omega} \Delta u \Delta \phi-\alpha \nabla u \nabla \phi=\lambda \int_{\Omega} u \phi \quad \forall \phi \in H^{2}(\Omega) \cap H_{0}^{1}(\Omega)
$$

the only difference between this and (2.1) being the ambient space. In particular, comparing the variational characterization (2.2) of the eigenvalues of problem (1.1) with that of the eigenvalues of the Navier problem (4.1),

$$
\lambda_{k}^{N}(\Omega, \alpha)=\min _{\substack{V \subset H^{2}(\Omega) \cap H_{0}^{1}(\Omega) \\ \operatorname{dim} V=k}} \max _{\substack{0 \neq u \in V \\ 0}} \frac{\int_{\Omega}(\Delta u)^{2}-\alpha|\nabla u|^{2}}{\int_{\Omega} u^{2}},
$$

yields

$$
\lambda_{k}^{D}(\Omega, \alpha) \geq \lambda_{k}^{N}(\Omega, \alpha) \quad \forall k \in \mathbb{N}, \forall \alpha \in \mathbb{R} .
$$

Now we want to compute eigenfunctions and eigenvalues of the Navier problem (4.1). We can of course proceed as for the Dirichlet case in section 2. However, we observe that we can modify the problem as follows:

$$
\begin{cases}\Delta^{2} u+\alpha \Delta u+\frac{\alpha^{2}}{4} u=\left(\lambda+\frac{\alpha^{2}}{4}\right) u & \text { in } \Omega \\ u=\Delta u+\frac{\alpha}{2} u=0 & \text { on } \partial \Omega\end{cases}
$$

which tells us immediately that if the domain has the cone property, the Navier operator in (4.4) is the square of the translated Dirichlet Laplace operator $\Delta+\frac{\alpha}{2}$ (cf. [16]). In particular, if we denote by $\gamma_{k}$ the $k$ th eigenvalue of the Dirichlet Laplacian (3.2), we get that the spectrum of (4.1) is given by

$$
\left\{\gamma_{k}^{2}(\Omega)-\alpha \gamma_{k}(\Omega)\right\}_{k}
$$

for any $\alpha \in \mathbb{R}$ and for any (smooth enough) domain $\Omega$. We remark that, for $\alpha<0$ (actually, for $\alpha<2 \gamma_{1}$ ), we have

$$
\lambda_{k}^{N}(\alpha)=\gamma_{k}^{2}-\alpha \gamma_{k}
$$

for any $k$, while on the other hand we actually have intersections of the branches (the intersection points will depend on $\Omega$ ). However, we can still say that

$$
\lambda_{1}^{N}(\alpha)=\min _{k}\left\{\gamma_{k}^{2}-\alpha \gamma_{k}\right\}=\min _{k}\left\{\left(\gamma_{k}^{2}-\frac{\alpha}{2}\right)^{2}\right\}-\frac{\alpha^{2}}{4} \geq-\frac{\alpha^{2}}{4},
$$

that is,

$$
\lambda_{k}^{D}(\alpha) \geq \lambda_{k}^{N}(\alpha) \geq \lambda_{1}^{N}(\alpha) \geq-\frac{\alpha^{2}}{4} \quad \forall \alpha \in \mathbb{R} .
$$


THEOREM 4.1. Let $\Omega$ be a bounded open set in $\mathbb{R}^{N}$ with the cone property. Then, for any $k \in \mathbb{N}$,

$$
\lambda_{k}^{N}(\alpha)=-\frac{\alpha^{2}}{4}+\mathrm{o}\left(\alpha^{2}\right)
$$

as $\alpha \rightarrow+\infty$. Moreover,

$$
\lambda_{1}^{N}(\alpha)=-\frac{\alpha^{2}}{4}+\mathrm{o}(\alpha)
$$

as $\alpha \rightarrow+\infty$.

Proof. Equality (4.7) easily follows from the inequality chain (4.6) coupled with the asymptotic expansion (1.3).

As for (4.8), we first observe that

$$
\lambda_{1}^{N}(\alpha)=\gamma_{k}^{2}-\alpha \gamma_{k} \quad \text { for } \gamma_{k-1}+\gamma_{k} \leq \alpha \leq \gamma_{k}+\gamma_{k+1},
$$

and for the choice $\alpha=2 \gamma_{k}$ we have

$$
\lambda_{1}^{N}(\alpha)=-\gamma_{k}^{2}=-\frac{\alpha^{2}}{4} .
$$

This alone is not enough to prove the asymptotic behavior. However, we know that $\lambda_{1}^{N}(\alpha)$ is a polygonal line and that each and every segment is tangent to the asymptotic curve (thanks to (4.9)). It is thus enough to show that the vertices have the same asymptotic behavior, i.e., the points $\alpha=\gamma_{k}+\gamma_{k+1}$ for which

$$
\lambda_{1}^{N}(\alpha)=-\gamma_{k} \gamma_{k+1}
$$

or, equivalently,

$$
\lambda_{1}^{N}(\alpha)-\frac{\alpha^{2}}{4}=\frac{\left(\gamma_{k+1}-\gamma_{k}\right)^{2}}{4}
$$

therefore, we have to show that

$$
\frac{\left(\gamma_{k+1}-\gamma_{k}\right)^{2}}{\gamma_{k+1}+\gamma_{k}} \rightarrow 0 \quad \text { as } k \rightarrow \infty .
$$

To this end, we recall the Weyl asymptotics for the Dirichlet eigenvalue problem for the Laplacian (3.2), namely

$$
\gamma_{k}=C_{1} k^{\frac{2}{N}}+C_{2} k^{\frac{1}{N}}+\mathrm{o}\left(k^{\frac{1}{N}}\right) \quad \text { as } k \rightarrow \infty
$$

where $C_{1}$ and $C_{2}$ are (known) constants depending only on $\Omega$ and the dimension $N$. From the binomial Taylor expansion

$$
(k+1)^{\delta}=k^{\delta}+\delta k^{\delta-1}+\mathrm{o}\left(k^{\delta-1}\right) \quad \text { as } k \rightarrow \infty,
$$

we have

$$
\frac{\left(\gamma_{k+1}-\gamma_{k}\right)^{2}}{\gamma_{k+1}+\gamma_{k}}=\frac{\left(\frac{2 C_{1}}{N} k^{\frac{2}{N}-1}+\frac{C_{2}}{N} k^{\frac{1}{N}-1}+\mathrm{o}\left(k^{\frac{1}{N}}\right)\right)^{2}}{2 C_{1} k^{\frac{2}{N}}+\mathrm{o}\left(k^{\frac{2}{N}}\right)},
$$

which clearly goes to zero for $N$ larger than one. 
Remark 4.2. If the domain is not bounded, it is still possible to prove (4.8) without using the asymptotics (1.3) while following the same strategy we used in the previous proof. In particular, in order to get the term $-\frac{\alpha^{2}}{4}$, it suffices to show that

$$
\frac{\left(\gamma_{k}+\gamma_{k+1}\right)^{2}}{\gamma_{k} \gamma_{k+1}} \rightarrow 4 \quad \text { as } k \rightarrow \infty
$$

which follows from the equality

$$
\frac{\left(\gamma_{k}+\gamma_{k+1}\right)^{2}}{\gamma_{k} \gamma_{k+1}}=\frac{\gamma_{k}}{\gamma_{k+1}}+\frac{\gamma_{k+1}}{\gamma_{k}}+2
$$

and the fact that the ratio of consecutive eigenvalues converges to 1 , thanks to Weyl's asymptotics (4.11).

Also, it is clear from (4.12) that the term o $(\alpha)$ in (4.8) is sharp since a different exponent in the denominator in the limit (4.10) would not go to zero as $k \rightarrow \infty$.

Remark 4.3. We observe that the behavior of the clamped plate problem (1.1) and that of the Navier problem (4.1) are substantially different. On the one hand, from the asymptotics (3.10) we have that the branches of eigenvalues of the clamped plate problem (1.1) will stop intersecting for some sufficiently large value of $\alpha$, at least in the case of balls where the parameters $t$ and $k$ provide a clear ordering of the branches, so that it is in principle possible to see which branch will eventually be the $k$ th eigenvalue. On the other hand, we know a priori that the branches of eigenvalues of the Navier problem (4.1) will have an infinite number of intersections, making it quite complicated to decide which is the $k$ th eigenvalue. In particular, the knowledge of the behavior of each individual branch does not provide sufficient information on the asymptotics of the eigenvalues. Similarly, even though the eigenspaces do not depend on $\alpha$, that associated with the $k$ th eigenvalue will keep on changing, creating a strange phenomenon of nonconvergence.

5. Shape derivatives. We will now consider the problem of finding extremal domains for the $k$ th eigenvalue of problem (1.1), namely the following.

Problem 1. Determine

$$
\lambda_{k}^{*}(\alpha)=\inf _{\Omega \subset \mathbb{R}^{n}}\left\{\lambda_{k}(\Omega, \alpha):|\Omega|=1\right\}
$$

We observe that proving existence for Problem 1 within a specific class of domains can be quite difficult and, to the best of our knowledge, there are no results available in general. To gauge the difficulties involved, we refer the reader to [7] for a survey on existence results for the Laplacian case, for which it is still not known whether existence holds within the class of open sets.

We will focus now on Problem 1 with $k=1$. We begin by deriving the formula for the Hadamard shape derivative of an eigenvalue of (1.1). Note that the formula in the case $\alpha=0$ was already derived in a general setting and for multiple eigenvalues; see $[10,24]$. We also refer the reader to [9] and the references therein for a complete discussion on Hadamard formulas for the biharmonic operator, also in the case $\alpha \neq 0$. Nevertheless, for the sake of simplicity we show here how to derive it in our specific case.

Consider an application $\Psi(t)$ such that $\Psi: t \in\left[0, T\left[\rightarrow W^{1, \infty}\left(\mathbb{R}^{N}, \mathbb{R}^{N}\right)\right.\right.$ is differentiable at 0 with $\Psi(0)=I, \Psi^{\prime}(0)=V$, where $W^{1, \infty}\left(\mathbb{R}^{N}, \mathbb{R}^{N}\right)$ is the set of bounded 
Lipschitz maps from $\mathbb{R}^{N}$ into itself, $I$ is the identity, and $V$ is a given deformation field.

We will use the notation $\Omega_{t}=\Psi(t)(\Omega)$ for a given set $\Omega, \lambda_{n}(t):=\lambda_{n}\left(\Omega_{t}, \alpha\right), u_{t}$ is an associated eigenfunction with unitary $L^{2}$ norm, and $u^{\prime}$ will denote the derivative of $u_{t}$ at $t=0$. Moreover, we assume that $\lambda_{n}(0)$ is simple.

It is well known (see, e.g., [13]) that if we define

$$
J(t)=\int_{\Omega_{t}} y(t, x) d x
$$

for some function $y$, then the Hadamard shape derivative is given by

$$
J^{\prime}(0)=\int_{\Omega} \frac{\partial y}{\partial t}(0, x) d x+\int_{\partial \Omega} y(0, x) V \cdot \nu d s_{x} .
$$

As a consequence, we have the following.

TheOREM 5.1. Let $\Omega$ be a bounded open set of class $C^{4}$. The Hadamard shape derivative for a simple eigenvalue $\lambda$ of problem (1.1) with corresponding eigenfunction $u$ is given by

$$
\lambda^{\prime}(0)=-\int_{\partial \Omega}\left(\frac{\partial^{2} u}{\partial \nu^{2}}\right)^{2} V \cdot \nu d s_{x}
$$

Proof. We have

$$
\lambda(t)=\int_{\Omega_{t}}\left(\Delta u_{t}\right)^{2}-\alpha\left|\nabla u_{t}\right|^{2} d x
$$

and the eigenfunction is normalized:

$$
\int_{\Omega_{t}} u_{t}^{2} d x=1
$$

The function $u^{\prime}$ can be calculated by solving the following boundary value problem (cf. $[17,18])$ :

$$
\begin{cases}\Delta^{2} u^{\prime}+\alpha \Delta u^{\prime}=\lambda^{\prime} u+\lambda u^{\prime} & \text { in } \Omega \\ u^{\prime}=0 & \text { on } \partial \Omega \\ \frac{\partial u^{\prime}}{\partial \nu}=-\frac{\partial^{2} u}{\partial \nu^{2}}(V \cdot \nu) & \text { on } \partial \Omega \\ \int_{\Omega} u u^{\prime} d x=0 . & \end{cases}
$$

Since the case $\alpha=0$ can be recovered from [24] (and can be done similarly to what follows), we assume $\alpha \neq 0$ and the eigenvalue equation can be written as

$$
\Delta u=\frac{\lambda u}{\alpha}-\frac{\Delta^{2} u}{\alpha}
$$


so that we have

$$
\begin{aligned}
\int_{\Omega} \nabla u \nabla u^{\prime} d x & =\int_{\partial \Omega} u^{\prime} \frac{\partial u}{\partial \nu} d s_{x}-\int_{\Omega} u^{\prime} \Delta u d x \\
& =-\int_{\Omega} u^{\prime}\left(\frac{\lambda u}{\alpha}-\frac{\Delta^{2} u}{\alpha}\right) d x \\
& =\frac{1}{\alpha}\left(\int_{\partial \Omega} u^{\prime} \frac{\partial(\Delta u)}{\partial \nu} d s_{x}-\int_{\Omega} \nabla u^{\prime} \nabla(\Delta u)\right) d x \\
& =-\frac{1}{\alpha}\left(\int_{\partial \Omega} \Delta u \frac{\partial u^{\prime}}{\partial \nu} d s_{x}-\int_{\Omega} \Delta u \Delta u^{\prime} d x\right) \\
& =-\frac{1}{\alpha} \int_{\partial \Omega} \Delta u\left(-\frac{\partial^{2} u}{\partial \nu^{2}}\right) V \cdot \nu d s_{x}+\frac{1}{\alpha} \int_{\Omega} \Delta u \Delta u^{\prime} d x \\
& =\frac{1}{\alpha} \int_{\partial \Omega} \Delta u\left(\frac{\partial^{2} u}{\partial \nu^{2}}\right) V \cdot \nu d s_{x}+\frac{1}{\alpha} \int_{\Omega} \Delta u \Delta u^{\prime} d x
\end{aligned}
$$

Applying now formula (5.1) to (5.3) and using (5.6), we obtain

$$
\begin{aligned}
\lambda^{\prime}(0) & =2 \int_{\Omega} \Delta u \Delta u^{\prime}-\alpha \nabla u \nabla u^{\prime} d x+\int_{\partial \Omega}(\Delta u)^{2} V \cdot \nu d s_{x} \\
& =2 \int_{\Omega} \Delta u \Delta u^{\prime} d x-2 \alpha \int_{\Omega} \nabla u \nabla u^{\prime} d x+\int_{\partial \Omega}(\Delta u)^{2} V \cdot \nu d s_{x} \\
& =2 \int_{\Omega} \Delta u \Delta u^{\prime} d x+2 \int_{\partial \Omega} \Delta u\left(-\frac{\partial^{2} u}{\partial \nu^{2}}\right) V \cdot \nu d s_{x} \\
& -2 \int_{\Omega} \Delta u \Delta u^{\prime} d x+\int_{\partial \Omega}(\Delta u)^{2} V \cdot \nu d s_{x} \\
& =\int_{\partial \Omega}\left(-2 \frac{\partial^{2} u}{\partial \nu^{2}} \Delta u+(\Delta u)^{2}\right) V \cdot \nu d s_{x} .
\end{aligned}
$$

The proof is concluded once we observe that $u \in H^{4}(\Omega)$ (cf. [16]), and since $u=\frac{\partial u}{\partial \nu}=0$ on $\partial \Omega$, we have that

$$
\Delta u=\frac{\partial^{2} u}{\partial \nu^{2}} \quad \text { on } \partial \Omega
$$

Remark 5.2. Using formula (5.2), we may try to attack Problem 1 via the Lagrange Multiplier Theorem. Since the constraint here is $|\Omega|=1$, we obtain the following condition:

$$
\frac{\partial^{2} u}{\partial \nu^{2}}=\text { constant } \quad \text { on } \partial \Omega
$$

Note that condition (5.7) then has to be added to problem (1.1), yielding an overdetermined problem resembling the Serrin problem (see [27]). However, problem (1.1) coupled with condition (5.7) is a more difficult problem, and the only partial result available in the literature can be found in [12].

It is worth observing that solving the overdetermined problem (1.1), (5.7) is not equivalent to solving Problem 1: in fact, the former provides just a critical point, which may be only a local minimizer, or even a local maximizer. Interestingly enough, though, eigenfunctions on the ball always satisfy condition (5.7). For a more detailed analysis of this fact, we refer the reader to $[9,10]$. 


\section{Numerical methods.}

6.1. Numerical solution of the eigenvalue problem. In this section, we will describe a numerical method for solving (1.1).

A fundamental solution $\Phi_{\lambda}$ of the partial differential equation of the eigenvalue problem (1.1) is given by (see, e.g., [20])

$$
\Phi_{\lambda}(x)=\frac{i\left(H_{0}^{(1)}\left(i \sqrt{\frac{1}{2}\left(\sqrt{\alpha^{2}+4 \lambda}-\alpha\right)}|x|\right)-H_{0}^{(1)}\left(\sqrt{\frac{1}{2}\left(\sqrt{\alpha^{2}+4 \lambda}+\alpha\right)}|x|\right)\right)}{4 \sqrt{\alpha^{2}+4 \lambda}},
$$

where $H_{0}^{(1)}$ is a Hankel function of the first kind.

We will consider particular solutions of the partial differential equation of the eigenvalue problem (1.1) by defining the boundary integral operators (for $x \in \Omega$ )

$$
u(x)=\int_{\hat{\Gamma}} \Phi_{\lambda}(x-y) \varphi(y) d s_{y}+\int_{\hat{\Gamma}} \partial_{\nu_{y}} \Phi_{\lambda}(x-y) \psi(y) d s_{y},
$$

where $\hat{\Gamma}$ is an artificial boundary that surrounds $\partial \Omega$ (see, e.g., $[1,3]$ ), and $\varphi$ and $\psi$ are densities. The numerical approximation of an arbitrary solution of the partial differential equation of the eigenvalue problem (1.1) can be justified by density results; see, e.g., $[1,2]$. Moreover, we will assume that $\hat{\Gamma}$ does not intersect $\bar{\Omega}$. Thus, we can discretize the boundary integral operators by considering the linear combinations

$$
u_{m}(x)=\sum_{j=1}^{m} \alpha_{m, j} \Phi_{\lambda}\left(x-y_{m, j}\right)+\sum_{j=1}^{m} \beta_{m, j} \partial_{\nu_{y_{m}, j}} \Phi_{\lambda}\left(x-y_{m, j}\right)
$$

where $y_{m, j}$ are some points on $\hat{\Gamma}$. Note that the functions $u_{m}$ are particular solutions of the partial differential equation involved in the eigenvalue problem (1.1) and the coefficients can be determined by fitting the boundary conditions of the problem.

We consider some collocation points $x_{1}, \ldots, x_{m}$, (almost) uniformly distributed on $\partial \Omega$, and impose the boundary conditions of the problem which leads to the $(2 m) \times(2 m)$ system

$(6.3)$

$$
\left\{\begin{array}{l}
0=u_{m}\left(x_{i}\right)=\sum_{j=1}^{m} \alpha_{m, j} \Phi_{\lambda}\left(x_{i}-y_{m, j}\right)+\sum_{j=1}^{m} \beta_{m, j} \partial_{\nu_{y_{m}, j}} \Phi_{\lambda}\left(x_{i}-y_{m, j}\right), \\
0=\partial_{\nu_{x_{i}}} u_{m}\left(x_{i}\right)=\sum_{j=1}^{m} \alpha_{m, j} \partial_{\nu_{x_{i}}} \Phi_{\lambda}\left(x_{i}-y_{m, j}\right)+\sum_{j=1}^{m} \beta_{m, j} \partial_{\nu_{x_{i}}} \partial_{\nu_{y_{m}, j}} \Phi_{\lambda}\left(x_{i}-y_{m, j}\right) .
\end{array}\right.
$$

We will consider the choice for source points $y_{m, j}$ described in [1], assume that $\nu_{y_{m, j}}=\nu_{x_{j}}$, and denote this vector simply by $\nu_{j}$. Using the notation $d_{i, j}=x_{i}-y_{m, j}$, the system (6.3) can be rewritten as

$$
\left\{\begin{array}{l}
0=\sum_{j=1}^{m} \alpha_{m, j} \Phi_{\lambda}\left(d_{i, j}\right)+\sum_{j=1}^{m} \beta_{m, j}\left(\nu_{j} \cdot \nabla \Phi_{\lambda}\left(d_{i, j}\right)\right) \\
0=\sum_{j=1}^{m} \alpha_{m, j}\left(\nu_{i} \cdot \nabla \Phi_{\lambda}\left(d_{i, j}\right)\right)+\sum_{j=1}^{m} \beta_{m, j}\left(\nu_{i} \cdot \nabla\left(\nu_{j} \cdot \nabla \Phi_{\lambda}\left(d_{i, j}\right)\right)\right) .
\end{array}\right.
$$

The approximations of the eigenvalues can be calculated by adapting the BetckeTrefethen method (see [6]) to this context. We consider $p$ points $z_{1}, z_{2}, \ldots, z_{p}$, randomly chosen in $\Omega$, and define the following six blocks: 


$$
\begin{aligned}
A(\lambda) & =\left[\Phi_{\lambda}\left(d_{i, j}\right)\right]_{m \times m}, & B(\lambda) & =\left[\nu_{j} \cdot \nabla \Phi_{\lambda}\left(d_{i, j}\right)\right]_{m \times m}, \\
C(\lambda) & =\left[\nu_{i} \cdot \nabla \Phi_{\lambda}\left(d_{i, j}\right)\right]_{m \times m}, & D(\lambda) & =\left[\nu_{i} \cdot \nabla\left(\nu_{j} \cdot \nabla \Phi_{\lambda}\left(d_{i, j}\right)\right)\right]_{m \times m}, \\
E(\lambda) & =\left[\Phi_{\lambda}\left(\tilde{d}_{i, j}\right)\right]_{p \times m}, & F(\lambda) & =\left[\nu_{j} \cdot \nabla \Phi_{\lambda}\left(\tilde{d}_{i, j}\right)\right]_{p \times m},
\end{aligned}
$$

where $\tilde{d}_{i, j}=z_{i}-y_{m, j}$. Then we define the matrix

$$
\mathbf{M}(\lambda)=\left[\begin{array}{ll}
A(\lambda) & B(\lambda) \\
C(\lambda) & D(\lambda) \\
E(\lambda) & F(\lambda)
\end{array}\right]
$$

compute the QR decomposition of $\mathbf{M}(\lambda)$, and calculate the minimal eigenvalue of the first $(2 m) \times(2 m)$ block of the matrix $\mathbf{M}(\lambda)$ that will be denoted by $\sigma_{1}(\lambda)$. The approximations for the eigenvalues of problem (1.1) are the values $\lambda$, for which $\sigma_{1}(\lambda) \approx$ 0 .

6.2. Numerical shape optimization. In this section, we will consider Problem 1 among general simply connected planar domains, whose boundary can be parametrized by

$$
\partial \Omega=\left\{\left(\Gamma_{1}(t), \Gamma_{2}(t)\right): t \in[0,2 \pi[\}\right.
$$

for some continuous and $(2 \pi)$-periodic functions $\Gamma_{1}$ and $\Gamma_{2}$. We will consider the (truncated) Fourier expansions

$$
\Gamma_{1}(t) \approx \gamma_{1}(t)=\sum_{j=0}^{P} a_{j}^{(1)} \cos (j t)+\sum_{j=1}^{P} b_{j}^{(1)} \sin (j t)
$$

and

$$
\Gamma_{2}(t) \approx \gamma_{2}(t)=\sum_{j=0}^{P} a_{j}^{(2)} \cos (j t)+\sum_{j=1}^{P} b_{j}^{(2)} \sin (j t)
$$

for a sufficiently large $P \in \mathbb{N}$, and the optimization procedure consists in finding optimal coefficients $a_{j}^{(1)}, b_{j}^{(1)}, a_{j}^{(2)}, b_{j}^{(2)}$. The optimization is performed by a gradienttype method, using the Hadamard shape derivative given by Theorem 5.1 to calculate the derivative of the eigenvalue with respect to perturbations of the coefficients $a_{j}^{(1)}$, $b_{j}^{(1)}, a_{j}^{(2)}, b_{j}^{(2)}$.

6.3. Numerical results. In this section, we present the main results that we gathered with our numerical procedure for solving Problem 1.

As was mentioned in the Introduction, each of the eigenvalue curves $\lambda_{k}(\alpha)$ is made up of analytic eigenvalue branches which intersect each other. We illustrate this fact in Figure 1. As was shown in Theorem 1.1, all the eigenvalues have the following asymptotic behavior:

$$
\lambda_{k}(\Omega, \alpha)=-\frac{\alpha^{2}}{4}+\mathrm{o}\left(\alpha^{2}\right)
$$

Thus, in order to produce more convenient pictures, instead of plotting the first eigenvalues as functions of $\alpha$, we will extract the first term of the expansion, which is the same for all eigenvalues; i.e., in Figure 1, we plot the quantities

$$
\lambda_{k}(\Omega, \alpha)+\frac{\alpha^{2}}{4}, \quad k=1,2, \ldots, 10
$$


for a disk of unit area and similar results for an ellipse with unit area and eccentricity equal to $\sqrt{3} / 2$.

Figure 2 shows the curve of the quantity $\lambda_{1}^{*}(\alpha)+\frac{\alpha^{2}}{4}$. We can observe several branches corresponding to different types of minimizers. Some of them, obtained for $\alpha=110,170,230,400$, are plotted in Figure 3. The optimal eigenvalue $\lambda_{1}^{*}(\alpha)$ is the minimum among the values obtained for all the branches. We calculated the critical value of $\alpha$, which is the maximal value of $\alpha$ for which the ball is the minimizer, and obtained $\alpha^{\star} \approx 102.23$. In [4], it was proved that the ball is the minimizer for $\alpha \in[0, a]$ and for some $a<\Lambda$, where $\Lambda=\pi j_{1,1}^{2} \approx 12.0377$ is the first buckling eigenvalue of the disk with unit area. Our numerical results suggest that actually the result may be true for a larger range of values of $\alpha$, and we conjecture that the ball is the minimizer for $\alpha \in\left[0, \alpha^{\star}\right]$. On the other hand, we have numerical evidence to support the conjecture that for $\alpha>\alpha^{\star}$ the ball is no longer the minimizer. For instance, for $\alpha=110$, the first eigenvalue of the ball of unit area can be directly calculated by solving (2.9) and is equal to $-1622.16613 \ldots$... In Table 1, we show some numerical approximations for the first eigenvalue of the minimizer that we obtained with our algorithm when $\alpha=110$, which is plotted in Figure 3 for different values of $m$. These results suggest that the first eigenvalue of this domain is equal to $-1786.35377 \ldots$, which is significantly smaller than the first eigenvalue of the disk.

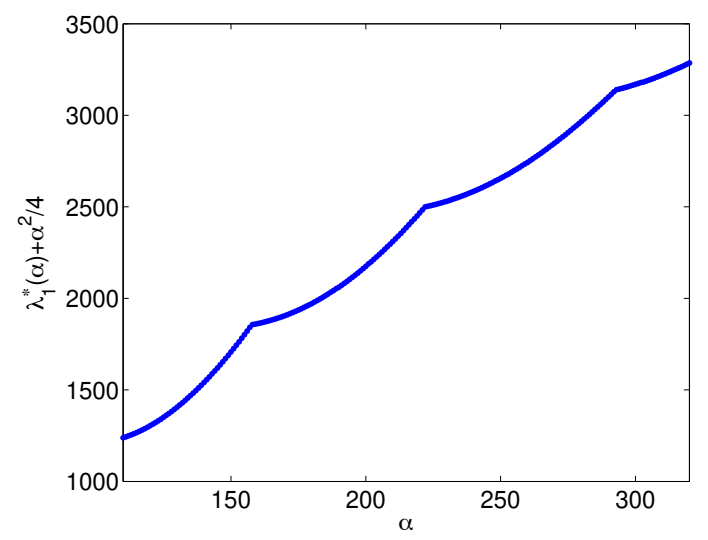

FIG. 2. The quantity $\lambda_{1}^{*}(\alpha)+\frac{\alpha^{2}}{4}$ for $\alpha \in[110,320]$.

In Figure 4, we plot the eigenfunctions associated with the first three eigenvalues of the optimizers of $\lambda_{1}$, obtained for $\alpha=110,170,230$. In this work, we considered just the optimization of the first eigenvalue. However, we observed that, besides the fact that the eigenfunction associated with the first eigenvalue changes sign, it also has a different number of nodal domains, depending on the parameter $\alpha$. Moreover, "similar" eigenfunctions appear associated with eigenvalues of different orders. For instance, the eigenfunction associated with the first eigenvalue for $\alpha=110$ is antisymmetric with respect to the first axis. However, the eigenfunction associated with the first eigenvalue for $\alpha=170$ is symmetric with respect to the first axis, and the first antisymmetric eigenfunction with respect to the first axis is associated not with the first eigenvalue but with the second eigenvalue.

Figure 5 shows a zoom of the boundary of the optimizer obtained numerically for $\alpha=110$ in a neighborhood of the re-entrant part of the boundary. Note that the 

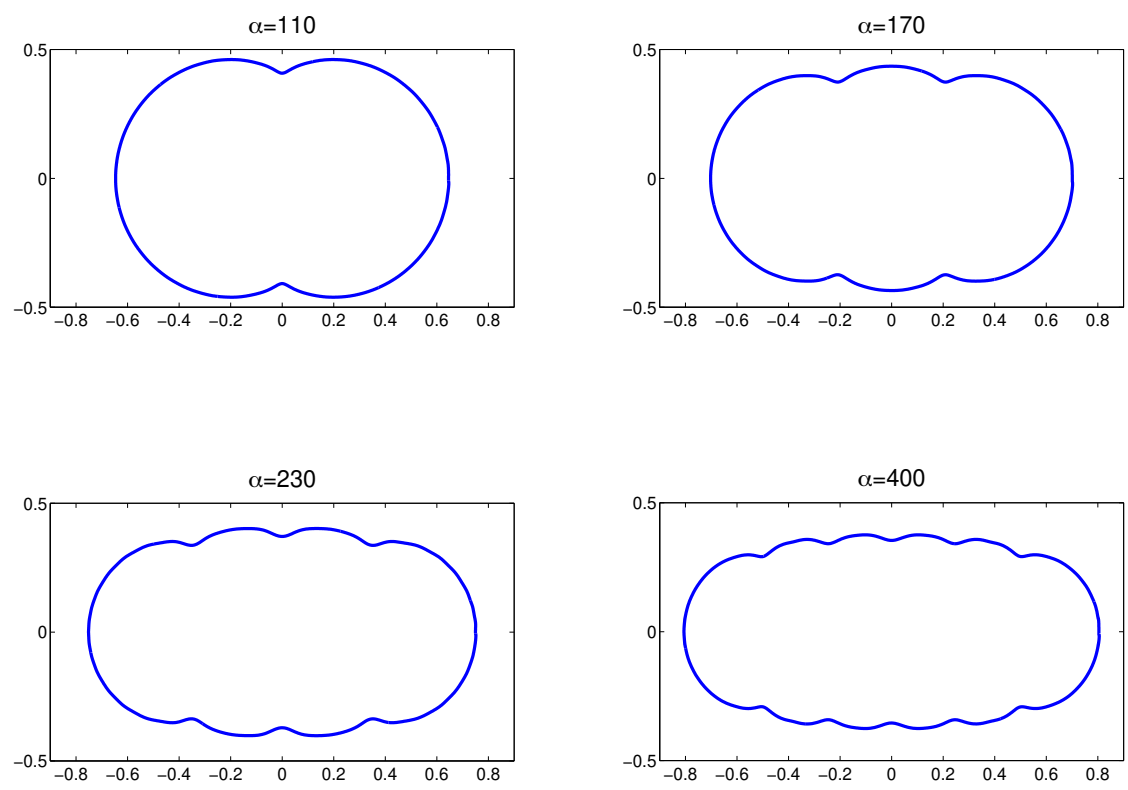

FIG. 3. Minimizers of $\lambda_{1}(\alpha)$ for $\alpha=110,170,230,400$.

TABLE 1

Numerical approximations obtained for the first eigenvalue of the minimizer when $\alpha=110$ for different values of $m$.

\begin{tabular}{|c|c|}
\hline$m$ & $\tilde{\lambda}_{1}$ \\
\hline 1000 & -1786.3537774 \\
\hline 1500 & -1786.3537779 \\
\hline 1800 & -1786.3537762 \\
\hline 2000 & -1786.3537753 \\
\hline
\end{tabular}

boundary of the domains considered in the optimization procedure was parameterized by a (truncated) Fourier expansion. In particular, the domains considered are always smooth and it is not clear how to obtain information on the regularity of the boundary of the optimizer from this. In particular, it is not possible to deduce whether this corresponds to a smooth boundary, a corner, or even a cusp.

Acknowledgments. Most of the research in this paper was carried out while the second author held a postdoctoral position at the University of Lisbon within the scope of this project. The second author is a member of the Gruppo Nazionale per l'Analisi Matematica, la Probabilità e le loro Applicazioni (GNAMPA) of the Istituto Nazionale di Alta Matematica (INdAM). 

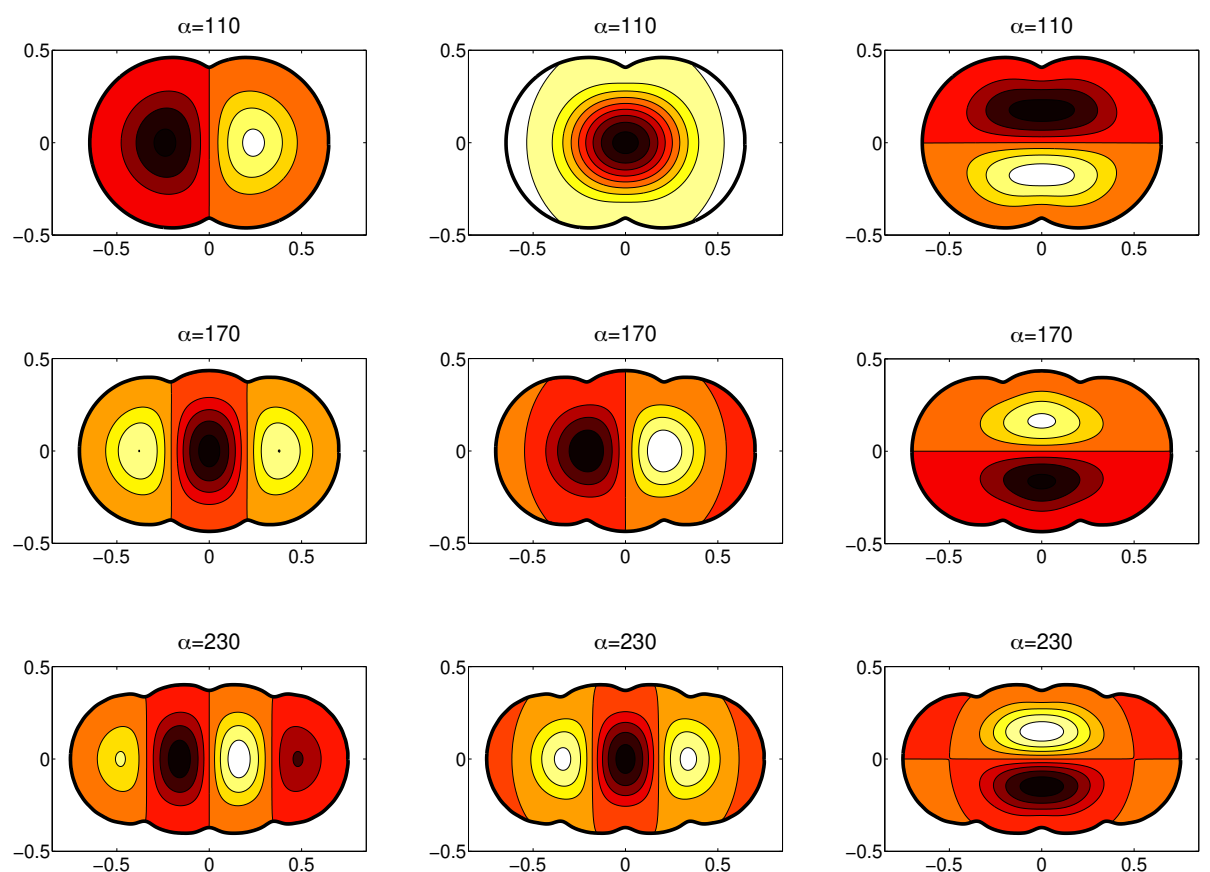

FIG. 4. Plots of the eigenfunctions associated with the first three eigenvalues of the optimizers of $\lambda_{1}$, obtained for $\alpha=110,170,230$.

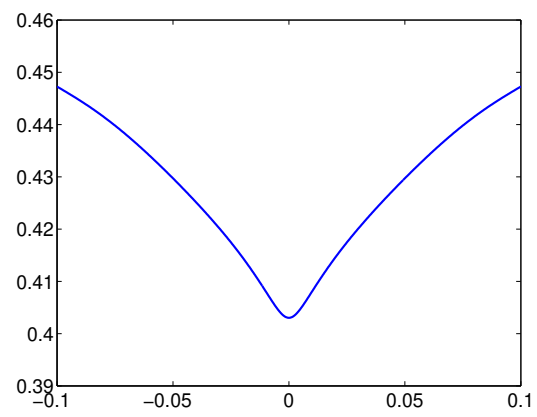

FIG. 5. Zoom of the boundary of the optimizer obtained for $\alpha=110$ close to the re-entrant region.

\section{REFERENCES}

[1] C. J. S. Alves And P. R. S. Antunes, The Method of Fundamental Solutions applied to the calculation of eigensolutions for 2 D plates, Internat. J. Numer. Methods Engrg., 77 (2009), pp. 177-194, https://doi.org/10.1002/nme.2404

[2] P. R. S. Antunes, On the buckling eigenvalue problem, J. Phys. A, 44 (2011), 215205.

[3] P. R. S. Antunes, Optimal bilaplacian eigenvalues, SIAM J. Control Optim., 52 (2014), pp. 2250-2260, https://doi.org/10.1137/130948860.

[4] M. S. Ashbaugh, R. Benguria, and R. Mahadevan, A Sharp Lower Bound for the First Eigenvalue of the Vibrating Clamped Plate Problem under Compression, preprint, 2018.

[5] M. S. Ashbaugh And R. D. Benguria, On Rayleigh's conjecture for the clamped plate and its 
generalization to three dimensions, Duke Math. J., 78 (1995), pp. 1-17, https://doi.org/ 10.1215/S0012-7094-95-07801-6.

[6] T. Betcke And L. N. Trefethen, Reviving the method of particular solutions, SIAM Rev., 47 (2005), pp. 469-491, https://doi.org/10.1137/S0036144503437336.

[7] D. Bucur, Existence results, in Shape Optimization and Spectral Theory, A. Henrot, ed., De Gruyter Open, Warsaw, Berlin, 2017, https://doi.org/10.1515/9783110550887-002.

[8] D. Bucur, P. Freitas, and J. B. Kennedy, The Robin problem, in Shape Optimization and Spectral Theory, A. Henrot, ed., De Gruyter Open, Warsaw, Berlin, 2017, https: //doi.org/10.1515/9783110550887-004.

[9] D. Buoso, Analyticity and criticality results for the eigenvalues of the biharmonic operator in Geometric Properties for Parabolic and Elliptic PDE's, Springer Proc. Math. Stat. 176, Springer, Cham, 2016, https://doi.org/10.1007/978-3-319-41538-3_5.

[10] D. Buoso And P. D. LAmBerti, Eigenvalues of polyharmonic operators on variable domains, ESAIM Control Optim. Calc. Var., 19 (2013), pp. 1225-1235, https://doi.org/10.1051/ $\operatorname{cocv} / 2013054$.

[11] L. M. Chasman And J. Chung, Spectrum of the free rod under tension and compression, Appl. Anal., 98 (2019), pp. 2145-2177, https://doi.org/10.1080/00036811.2018.1451639.

[12] R. Dalmasso, Un problème de symétrie pour une équation biharmonique, Ann. Fac. Sci. Toulouse Math. (5), 11 (1990), pp. 45-53.

[13] M. C. Delfour And J.-P. ZolÉsio, Shapes and Geometries: Analysis, Differential Calculus, and Optimization, Adv. Des. Control 4, SIAM, Philadelphia, 2001, https://doi.org/10. $1137 / 1.9780898719826$.

[14] L. S. FRANK, Coercive singular perturbations: Eigenvalue problems and bifurcation phenomena, Ann. Mat. Pura Appl. (4), 148 (1987), pp. 367-395, https://doi.org/10.1007/BF01774296.

[15] P. Freitas and R. Laugesen, From Neumann to Steklov and Beyond, via Robin: The Weinberger Way, preprint, https://arxiv.org/abs/1810.07461, 2018.

[16] F. Gazzola, H.-C. Grunau, And G. Sweers, Polyharmonic Boundary Value Problems. Positivity Preserving and Nonlinear Higher Order Elliptic Equations in Bounded Domains, Lecture Notes in Math. 1991, Springer-Verlag, Berlin, 2010, https://doi.org/10.1007/ 978-3-642-12245-3.

[17] P. Grinfeld, Hadamard's formula inside and out, J. Optim. Theory Appl., 146 (2010), pp. 654-690, https://doi.org/10.1007/s10957-010-9681-6.

[18] A. Henrot And M. Pierre, Variation et optimisation de formes. Une analyse géométrique, Math. Appl. (Berlin) 48, Springer, Berlin, 2005, https://doi.org/10.1007/3-540-37689-5.

19] B. Kawohl, H. A. Levine, And W. Velte, Buckling eigenvalues for a clamped plate embedded in an elastic medium and related questions, SIAM J. Math. Anal., 24 (1993), pp. 327-340, https://doi.org/10.1137/0524022.

[20] M. Kitahara, Boundary Integral Equation Methods in Eigenvalue Problems of Elastodynamics and Thin Plates, Elsevier, Amsterdam, 1985.

[21] A. Love, A Treatise on the Mathematical Theory of Elasticity, 4th ed., Dover, New York, 1944

[22] N. S. NAdirashvili, Rayleigh's conjecture on the principal frequency of the clamped plate, Arch. Rational Mech. Anal., 129 (1995), pp. 1-10, https://doi.org/10.1007/BF00375124.

[23] F. W. J. Olver, D. W. Lozier, R. F. Boisvert, and C. W. Clark, eds., NiSt Handbook of Mathematical Functions, Cambridge University Press, Cambridge, UK, 2010.

[24] J. H. Ortega And E. ZuAzuA, Generic simplicity of the spectrum and stabilization for a plate equation, SIAM J. Control Optim., 39 (2001), pp. 1585-1614, https://doi.org/10.1137/ S0363012900358483.

[25] K. Pankrashkin And N. Popoff, Mean curvature bounds and eigenvalues of Robin Laplacians, Calc. Var. Partial Differential Equations, 54 (2015), pp. 1947-1961, https://doi.org/10. 1007/s00526-015-0850-1.

[26] J. W. S. Rayleigh, The Theory of Sound, 2nd ed., Dover, New York, 1945.

[27] J. SerRin, A symmetry problem in potential theory, Arch. Rational Mech. Anal., 43 (1971), pp. 304-318, https://doi.org/10.1007/BF00250468. 